\title{
Simple Simulation for COVID-19 in Wuhan, Hubei, China to Predict Outbreak in Wuhan, the Initial Case in Wuhan, and the Epidemic in Japan as of 11 February, 2020
}

\author{
Junko Kurita1, Tamie Sugawara², Yoshiyuki Sugishita², Yasushi Ohkusa2* \\ ${ }^{1}$ Department of Nursing Collage of Nursing, Tokiwa University, Ibaraki, Japan \\ ${ }^{2}$ Infectious Disease Surveillance Center, National Institute of Infectious Diseases, Tokyo, Japan \\ Email: *ohkusa@nih.go.jp
}

How to cite this paper: Kurita, J., Sugawara, T., Sugishita, Y. and Ohkusa, Y. (2020) Simple Simulation for COVID-19 in Wuhan, Hubei, China to Predict Outbreak in Wuhan, the Initial Case in Wuhan, and the Epidemic in Japan as of 11 February, 2020. Journal of Biosciences and Medicines, 8 , 56-61.

https://doi.org/10.4236/jbm.2020.86006

Received: April 28, 2020

Accepted: May 29, 2020

Published: June 1, 2020

Copyright $\odot 2020$ by author(s) and Scientific Research Publishing Inc. This work is licensed under the Creative Commons Attribution International License (CC BY 4.0).

http://creativecommons.org/licenses/by/4.0/

\section{(c) (i) Open Access}

\begin{abstract}
Object: Prediction of the COVID-19 epidemic represents a matter of concern not only for public health or medicine but also for Earth's general population. This study predicts outbreaks in Wuhan and in Japan as of 11 February, 2020. Method: We applied a simple SIR model to data published by Hubei public health authorities. Moreover, into the model, we incorporate mild and asymptomatic cases from experiences of Japanese residents of Wuhan up to the outbreak. Finally, we predict an outbreak in Japan based on 10,000 iterations of a simulation conducted under the assumption of infected people including mild cases visiting Japan according to the estimated distribution of patients in Wuhan since the date on which the initial case occurred to the date when travel from Wuhan to Japan was suspended. Results: Results suggest the basic reproduction number, $R_{0}$, as 2.84 ; its $95 \%$ confidence interval (CI) was $[2.35,3.33]$. The peak is estimated to be reached on March 11. Its 95\% CI peak date is 29 February to 27 March. The 95\% CI peak date in Japan is 26 April to 2 May. The greatest number of patients at the peak with severe symptoms was estimated as 858.3 thousand. Discussion and Conclusion: Our obtained $R_{0}$ of 2.84 approximates an earlier estimate. We predicted the greatest number of patients at the peak with severe symptoms as 858.3 thousand in Japan. This number is $63 \%$ greater than the highest daily peak of influenza.
\end{abstract}

\section{Keywords}

COVID-19 Outbreak in Japan, SIR Model, Prediction, Mild Case, Asymptomatic Case 


\section{Introduction}

The initial case of COVID-19 was reported in Wuhan on 1 December, but human-to-human infection was not confirmed at that time [1]. Since 23 January, Wuhan city has blocked traffic. On 29 January, WHO declared a Public Health Emergency of International Concern. As of 16 February, WHO reported 51,857 laboratory-confirmed cases and 1666 cases with mortality [2]. The prediction of the COVID-19 epidemic represents an important concern not only for public health or medicine but also for Earth's general population.

Already, basic reproduction number, $R_{0}$, of COVID-19 was estimated in the very earliest stage of outbreak [3]. Nevertheless, its entire course of outbreak and its spread to other cities in China and other countries were not examined.

This study examines $R_{0}$ estimated by the Hubei public health authorities by using susceptible-infected-recovery (SIR) model [4] and predicts the outbreak course in Wuhan by application of a similar procedure to that used for 2009 pandemic influenza [4]. Especially, we incorporate mild cases or asymptomatic cases which were not reported in China, based on experiences of Japanese residents of Wuhan: that were 11 mild cases, and 2 asymptomatic cases among 962 persons. Finally, we assess the predicted outbreak course in Japan as of 11 February, 2020.

\section{Method}

We applied a simple SIR model to the data assuming the following conditions: the incubation period was 3 - 7 days with a uniform distribution [3]. We defined severe cases those who showed pneumonia and mild cases those who showed fever and respiratory symptoms but did not show pneumonia. Experience among Japanese people living in Wuhan until the outbreak provides information for mild cases because complete seroepidemiological surveillance was performed for them. During January 29-February 7, 2020, in total 962 Japanese returned to Japan from Wuhan. All had received a test to detect COVID-19; of them, 12 were found to be positive for COVID-19 [5]. Of those 12, 9 Japanese people had exhibited mild symptoms; the other 3 showed no symptom. Moreover, two Japanese residents of Wuhan exhibited severe symptoms: one died, although no fatal case was confirmed as COIVD-19 by testing. However, two Japanese residents of Wuhan with mild symptoms were refused re-entry to Japan even though they were not confirmed as infected. If one assumes that the Japanese fatal case in Wuhan and two rejected re-entrants were infected with COVID-19, then 2 severe cases, 11 mild cases, and 2 asymptomatic cases existed among Japanese residents of Wuhan. We apply these proportions to the simulation.

We assumed that the power of infectivity among severe patients and mild patients were equal, but in the asymptomatic cases, it was assumed to be half of symptomatic cases as influenza [6] [7] [8] [9] [10]. We searched $R_{0}$ to fit the number of patients during 20 January to 11 February in Wuhan assuming they were severe cases so as to minimize the sum of absolute values of discrepancy 
among the reported numbers and the fitted values. Before 20 January, the daily number of confirmed cases was not reported by Hubei public health authorities. After 11 February, case definitions of the infected patients were changed to include clinically diagnosed individuals to confirmed cases in addition to test-positive cases as before. We identified the confirmed cases during this period as severe cases: they include no mild or asymptomatic cases.

Its $95 \%$ confidence interval (CI) was estimated by the second derivative of $R_{0}$ to the sum of squared discrepancy among data. It was fitted during this period.

First, we estimated $R_{0}$. We also simulated data before January 19 to infer the initial appearance of a case in Wuhan. Then we estimated how many people were infected with mild symptoms at a date when the initial case occurred in Wuhan to the end of January, when travel from Wuhan to Japan was suspended. We presumed that, because of their illness, no severely ill patient would be allowed to return to Japan.

Finally, we predicted the outbreak in Japan through 10,000 iterations of bootstrapping under the assumption that infected people, including those with mild cases, visit Japan randomly according to an estimated distribution of patients in Wuhan and $R_{0}$ from the date when the initial case occurred to 22nd January when Wuhan city was blocked traffic. During this period, we assumed about 1.02 million from the number of Chinese including other than Wuhan came to Japan as experience in late of 2019 and early in 2019 [11].

\section{Results}

$R_{0}$ was estimated as 2.84 . Its $95 \%$ CI was [2.35, 3.33]. The predicted epidemic curve during 20 January through 11 February is portrayed in Figure 1. Figure 2 depicts the predicted entire epidemic curve in Wuhan. The peak was estimated as being reached on March 11 with 786.6 thousand newly diagnosed severe patients. The 95\% CI peak date was 29 February to 27 March, with respective maximum numbers of severe patients per day estimated respectively as 615.8 and 936 thousand. Working backward, we also estimate that the first initial severe case in Wuhan occurred on 12 December, 2019. Between 12 December and 31 January, 112.51 thousand cases with mild infection and 21.304 thousand asymptomatic cases were estimated during the same period because about two million Chinese were estimated as visiting. In all, 160.73 infected people with mild symptoms and 30.43 infected asymptomatic cases from Wuhan were estimated as visiting Japan. Results of 10,000 simulation iterations show a 95\% CI peak date in Japan as 31st March to 26th May. The number of patients with severe symptoms at the peak was estimated as 858.3 thousand if $R_{0}$ was estimated value.

\section{Discussion}

We estimate the simple SIR model including mild or asymptomatic cases that were not published from the Hubin public health authorities in China. Although 


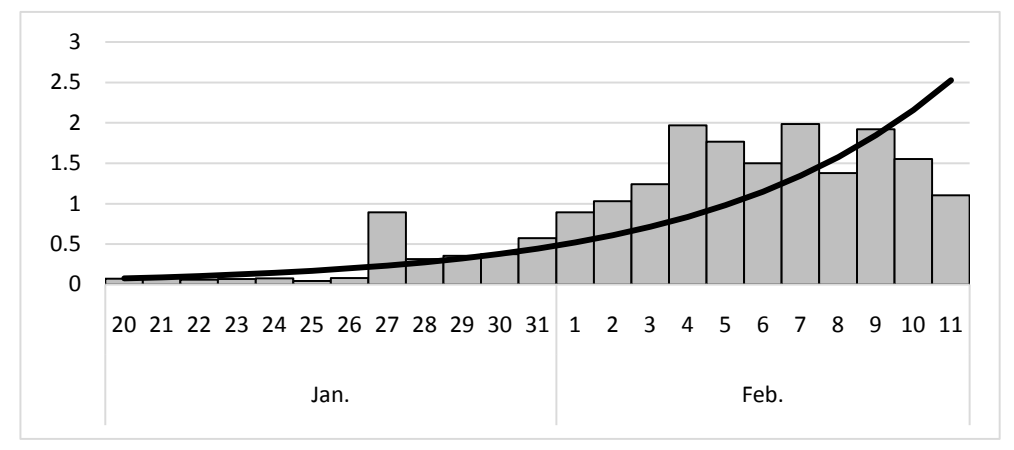

Figure 1. Epidemic curve of COVID-19 in Wuhan and fitted lines from 20 January to 11 February (number of patients in thousands). Note: Bars represent the epidemic curve in Wuhan reported from the Hubei public health authority. The line represents the fitted epidemic curve based on the estimated $R_{0}$.

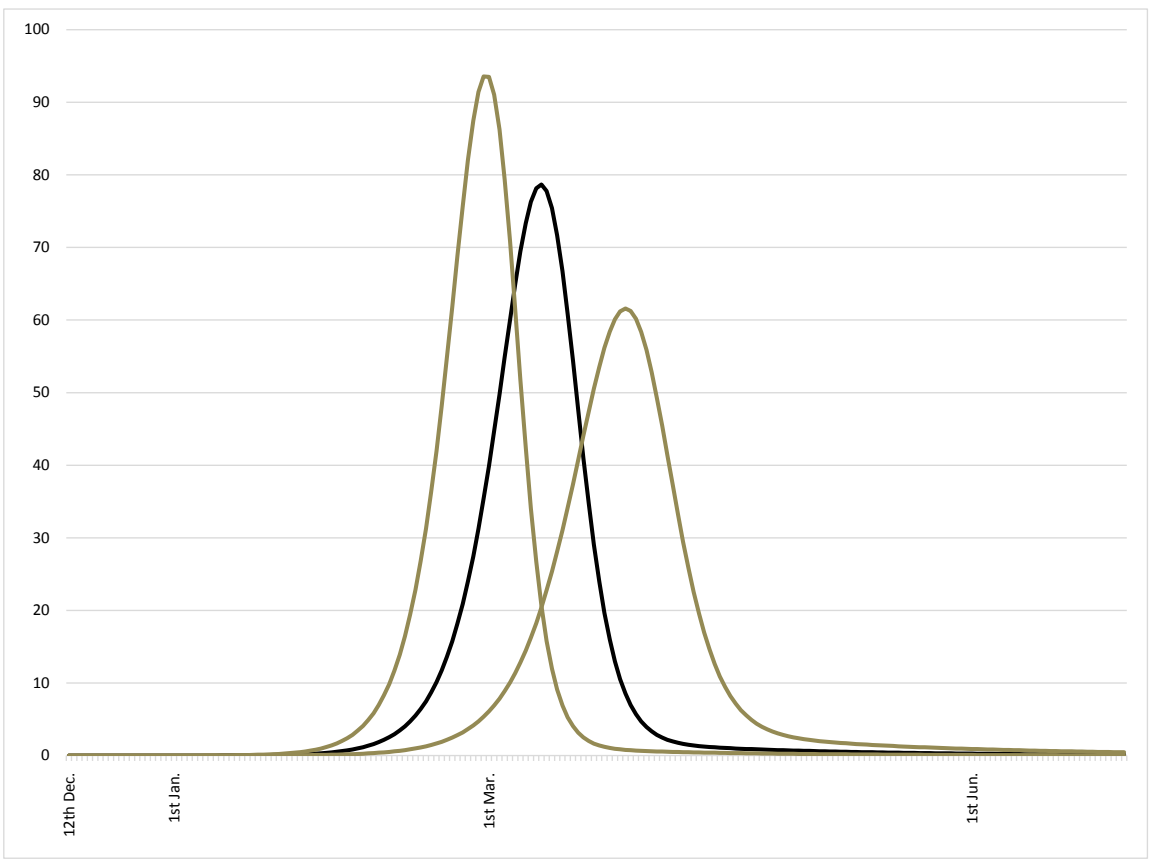

Figure 2. Predictive epidemic curve of COVID-19 in Wuhan and its $95 \%$ confidence interval. Note: The black line represents the epidemic curve based on the estimated $R_{0}$ of $2.84[2.35,3.33]$. Two gray lines represent the epidemic curves of the $95 \%$ confidence interval estimated $R_{0}$ as $[2.35,3.33]$.

the initial case was reported in Wuhan on1 December [1], we estimated the onset of the initial case with estimated power of infectiousness on 12 December, An earlier study [2] estimated $R_{0}$ for COVID-19 as $2.24-3.58$. Our obtained $R_{0}, 2.84$ was similar to the earlier reported one.

We assumed equal power of infectiousness by severe patients and mild patients. This assumption reflects the fact that mild patients usually do not change their behavior and contacts. For that reason, they can infect others effectively, although their volume virus shading might be smaller than that of severely ill patients. Conversely, virus shading among severe patients might be greater than 
that of mild patients. However, their activity would greatly decline, especially if they were hospitalized. Therefore, we cannot identify ex ante which type of patient has greater infectiousness.

We predicted the greatest number of patients at peak with severe symptoms as 858.3 thousand in Japan. This number was 58\% larger than the highest peak of influenza on any day since 2009, which was 15 January, 2019 [12]. The number of patients itself might be comparable, but typical symptoms of COVID-19 including pneumonia are much severer than those of influenza. Therefore, the severe COVID-19 patients are expected to be hospitalized, although most influenza patients in Japan never became hospital inpatients. Additional beds might also be needed, as they were necessary in Wuhan, if all of 858.3 thousand patients were hospitalized simultaneously. Preparation of beds, drugs, and respirators around the peak is expected to be necessary, although only 70 days remain before the peak is reached.

As portrayed in Figure 1, some spikes were apparent in the reported number of patients from the Hubei public health authorities. Therefore, we fitted $R_{0}$ to minimize the absolute deviation between the reported number of cases and fitted, instead of the squared residual. The number of confirmed cases we used was presumed to be affected by the test capacity or test performance for sensitivity and specificity. Therefore, the data which were used might not reflect the outbreak in Wuhan precisely. They might downwardly bias the estimate of $R_{0}$. Some studies have examined underreporting in China [2] [13], but we pointed out the possibility that, because they had not incorporated mild cases or asymptomatic cases into their model, they misunderstand them as underreporting of the confirmed cases in China.

Nevertheless, if some drug, vaccine or effective therapy were developed, the obtained results would eventually turn out to be an overestimation. We hope to realize development of such countermeasures. Even so, this primitive and simple simulation might contribute to planning that is capable of controlling the outbreak.

\section{Conclusion}

We predicted the peak of the outbreak in Wuhan as March 11; its 95\% CI peak was inferred as 29 February to 27 March. We also predicted the outbreak peak in Japan as occurring at about the end of April to early May. We hope that the obtained results will support public health authorities' decisions related to controlling.

\section{Conflicts of Interest}

The authors declare no conflicts of interest regarding the publication of this paper.

\section{References}

[1] Huang, C.L., Wang, Y.M., Li, X.W., Ren, L.L., Zhao, J.P., Hu, Y., Zhang, L., Fan, 
G.H., Xu, J.Y., Gu, X.Y., Cheng, Z.S., Yu, T., Xia, J., Wei, Y., Wu, W.J., Xie, X.L., Yin, W., Li, H., Liu, M., Xiao, Y., Gao, H., Guo, L., Xie, JG.., Wang, G.F., Jiang, R.M., Gao, Z.C., Jin, Q., Wang, J.W. and Cao, B. (2020) Clinical Features of Patients Infected with 2019 Novel Coronavirus in Wuhan, China. Lancet, 395, 497-506. https://doi.org/10.1016/S0140-6736(20)30183-5

[2] WHO (2020) Coronavirus Disease 2019 (COVID-19) Situation Report-27. https://www.who.int/docs/default-source/coronaviruse/situation-reports/20200216sitrep-27-covid-19.pdf?sfvrsn=78c0eb78_2

[3] Zhao, S., Lin, Q.Y., Ran, J.J., Musa, S.S., Yang, G.P., Wang, W.M., Lou, Y.J., Gao, D.Z., Yang, L., He, D.H. and Wang, M.H. (2020) Preliminary Estimation of the Basic Reproduction Number of Novel Coronavirus (2019-nCoV) in China, from 2019 to 2020: A Data-Driven Analysis in the Early Phase of the Outbreak. International Journal of Infectious Diseases, 92, 214-217. https://doi.org/10.1101/2020.01.23.916395

[4] Ohkusa, Y., Sugawara, T., Taniguchi, K. and Okabe, N. (2011) Real-Time Estimation and Prediction for Pandemic A/H1N1(2009) in Japan. Journal of Infection and Chemotherapy, 17, 468-472. https://doi.org/10.1007/s10156-010-0200-3

[5] Japan Ministry of Health, Labour and Welfare (2020) Press Releases. (In Japanese) https://www.mhlw.go.jp/stf/houdou/houdou_list_202001.html

[6] Ferguson, N.M., Cummings, D.A., Cauchemez, S., Fraser, C., Riley, S., Meeyai, A., et al. (2005) Strategies for Containing an Emerging Influenza Pandemic in Southeast Asia. Nature, 437, 209-214. https://doi.org/10.1038/nature04017

[7] Longini Jr., I.M., Nizam, A., Xu, S., Ungchusak, K., Hanshaoworakul, W., Cummings, D.A. and Halloran, M.E. (2005) Containing Pandemic Influenza at the Source. Science, 309, 1083-1087. https://doi.org/10.1126/science.1115717

[8] Germann, T.C., Kadau, L., Longini Jr., I.M. and Macken, C.A. (2006) Mitigation Strategies for Pandemic Influenza in the United States. Proceedings of the National Academy of Sciences of the United States of America, 103, 5935-5940. https://doi.org/10.1073/pnas.0601266103

[9] Ferguson, N.M., Cummings, D.A., Fraser, C., Cajka, J.C., Cooley, P.C. and Burke, D.S. (2006) Strategies for Mitigating an Influenza Pandemic. Nature, 442, 448-452. https://doi.org/10.1038/nature04795

[10] Ohkusa, Y. and Sugawara, T. (2009) Simulation Model of Pandemic Influenza in the Whole of Japan. Journal of Japanese Infectious Disease, 62, 98-106.

[11] Japan National Tourism Organization (2020) Trends in Foreign Visitors to Japan. (In Japanese) https://www.jnto.go.jp/jpn/statistics/visitor_trends/

[12] Prescription Surveillance. The Estimated Number of Patients Who Were Prescribed. (In Japanese) http://prescription.orca.med.or.jp/kanjyasuikei/

[13] Zhao, S., Musa, S.S., Lin, Q.Y., Ran, J.J., Yang, G.P., Wang, W.M., Lou, Y.J., Yang, L., Gao, D.Z., He, D.H. and Wang, M.H. (2020) Estimating the Unreported Number of Novel Coronavirus (2019-nCoV) Cases in China in the First Half of January 2020: A Data-Driven Modelling Analysis of the Early Outbreak. Journal of Clinical Medicine, 9, 388. https://doi.org/10.3390/jcm9020388 